\title{
Hot Magnetic Stars in Exotic Multiple Systems
}

\author{
Eugene Semenko ${ }^{1,2, *}$ \\ ${ }^{1}$ National Astronomical Research Institute of Thailand (NARIT), 260 Moo 4, T. Donkaew, A. Maerim, \\ Chiangmai, 50180 Thailand \\ ${ }^{2}$ Special Astrophysical Observatory of the Russian Academy of Sciences, Nizhnii Arkhyz, Russia, \\ 369167
}

\begin{abstract}
A strong and stable magnetic field covering the whole surface is a landmark of chemically peculiar or CP stars. To explain the origin of magnetic stars, several hypotheses were proposed, which depending on the mechanism and time of formation can be grouped into three main. The magnetic field may originate in the protostellar medium ("fossil field"), turbulent layers of a star ("dynamo" mechanism) or in the other environment. In either scenario, binary stars appear as a merit of its reliability.

Recent advances in observational astrophysics uncovered a bunch of new binary magnetic stars that were considered as rare before. We outline the results of studies of exotic binary and multiple systems with magnetic CP components: HD 6757, HD 34736, and HD 40759.
\end{abstract}

\section{Introduction}

Magnetic fields are ubiquitous in space. With the different scale and strength, the fields often take part in birth, life, and death of the stars. Objects with measurable magnetic field appear in all parts of the Hertzsprung-Russell diagram. In her review, L. Ferrario [1] counted different cases of magnetic objects, mostly heavier than two solar masses, and gave an overview of their possible evolutional models. These massive objects have varying age and possess fields ranging from about one gauss to more than thirty kilogauss. In the lower part of the Main Sequence, the dwarfs hold the fields, which are the strongest among the stars with convective envelopes. As an example, recent searches of magnetic fields in M-dwarfs have revealed the surface field of the order of several $\mathrm{kG}$ [2]. A weak, the order of gauss field was also found in some evolved objects far beyond the MS. The extensive list of references covering this problem can be found in an introduction to a paper by Mathias et al. [3] devoted to the long-term study of the magnetic field in Betelgeuse.

Magnetic fields of cool stars often are closely related to their activity. Famous solar spots are the most known example of such activity, but it can manifest itself in many other ways. There are cases of radio and X-ray emission attributed to the flares. Chromospheric flares are another way of presentation of the magnetic field. Until recently, it was believed that the activity on the Main Sequence is intrinsic to cool dwarfs and the hottest stars. In contrast, single intermediate-mass stars were considered as relatively quiet objects, even if they host a strong magnetic field. The concept has been changed with a discovery of radio emission

\footnotetext{
*e-mail: eugene@ @arit.or.th
} 
from hot B chemically peculiar stars. Now, five Bp-stars with coherent radio emission are known [4].

In the list of magnetic objects, chemically peculiar or CP stars take a special place. They occupy the upper part of the HR diagram in the range of the effective temperature from about 7000 to almost $25000 \mathrm{~K}$, and spread over the entire width of the Main Sequence. Three main hypotheses were proposed to explain the origin of stellar magnetic fields. The most evident one is a "turbulent dynamo" hypothesis $[5,6]$. This mechanism is responsible for the generation of the magnetic field in stars with strong convection envelopes like our Sun. In the case of CP-stars, dynamo might play an important role in the early stages of stellar evolution. Still, later, during the MS stage, its importance must decrease as the stellar atmospheres become mostly radiative. If the field originated in a deep convective core of a typical Ap/Bpstar, then its emerging time would exceed MS lifetime of the star.

Within the paradigm of the "fossil" field, one can explain a slow decay of the field on the MS. This hypothesis states that the field, which had been originated during the early stage of star formation, during the MS phase slowly dissipates [7, 8]. By default, the observed field takes its origin in the galactic seed field, but the hypothesis does not exclude other sources.

As a combination of the best of two previous hypotheses, the third one has been developed recently by Ferrario et al. [9]. This hypothesis, being concordant to the "fossil" theory, suggests that the seed magnetic field was formed in the process of the active mixing during the stellar merges. In this sense, it could be considered as a partial case of fossil field theory with alternative initial conditions. This scenario must not only explain the low fraction of magnetic CP-stars within the same spectral type but also make clear why is so small the number of close binaries with magnetic components.

Nowadays, there is no consensus in a problem of field origin, but observations mostly witness in favour of fossil nature of the field. At least such fields are observed during the MS stage. In either case, none of the three hypotheses can explain all diversity of the observed parameters of the magnetic CP-stars.

In this regard, binary and multiple stellar systems seem to be the natural test for the proper choice of the origin hypothesis. As the different scenarios predict the contrasting rates of binaries, varying orbital periods, and distinctive individual properties of the stars, it is crucial to study the systems where the components are of the same mass or anomalies.

\section{Chemically Peculiar Stars}

From the introduction, it becomes clear that in the Upper Main Sequence only chemically peculiar or CP stars could be recognised as magnetic objects. However, CP stars contain many different objects, which form the group of 10-15\% of MS stars showing abnormally enhanced or weakened lines of some specific elements in spectra. It was Preston [10] who divided all $\mathrm{CP}$ stars into four groups according to a dominating type of anomalies. The Astars, mostly with deficient $\mathrm{Ca}$ and $\mathrm{Sc}$ and overabundant heavy metals, fill the first group $\mathrm{CP} 1$. The second, CP2 group consists of A-stars with enhanced $\mathrm{Fe}, \mathrm{Cr}, \mathrm{Sr}$, and some rareearth elements. In the third group, Preston classified the stars with HgMn type of anomalies. The last group, CP4, contains the stars with abnormally weakened He lines in the spectra. It is worth to note here that the pairs $\mathrm{CP} 1-\mathrm{CP} 2$ and $\mathrm{CP} 3-\mathrm{CP} 4$ share the same range of effective temperatures, but there is the feature that suggests another combination. In total, about 500 stars in groups CP2 and CP4 (and some fraction of the stars with enhanced He lines which are out of this scheme) host strong and stable magnetic fields. Neither example of such field was found for metallic-line Am or HgMn stars. Thus, from lower to higher effective temperature, $\mathrm{CP} 2-\mathrm{CP} 4$ and, respectively, $\mathrm{CP} 1-\mathrm{CP} 3$ form two continuous sequences of magnetic and nonmagnetic stars. 
The magnetic field of $\mathrm{CP} 2$ and $\mathrm{CP} 4$ (or hereafter $\mathrm{mCP}$ ) stars ranges from hundreds of gauss to more than $30 \mathrm{kG}$, covers the whole surface, and remains stable on a timescale of decades. Measured characteristics of the field vary with a period of stellar axial rotation. Thus, the typical magnetic CP-star looks like a large rotating magnet. This feature differs mCP stars from cool dwarfs, even with a comparable value of their mean surface field. Magnetic Doppler Imaging [11], a unique technique that allows mapping of the surface of these stars, reveals simple field geometry with mostly dipolar structure combined with multipoles of higher orders in some cases [12]. The total number of CP-stars with the confirmed magnetic field is about 500 and continuously grows as the major programmes aimed at searches of such instances are carrying out [13].

Multiplicity is another key difference between the magnetic and non-magnetic groups of $\mathrm{CP}$ stars. From numerous studies, it is known that $\mathrm{CP} 1$ and $\mathrm{CP} 3$ stars tend to appear in binary and multiple stellar systems. The fraction of binaries, in this case, is close to 90\% [14]. Many of the stars are in spectroscopic binary systems, but there is also a significant fraction of $\mathrm{Am} / \mathrm{HgMn}$ in wide pairs [15]. Orbital periods of SB systems containing non-magnetic CP stars fall mostly between 1.2 and 100 days.

In contrast, mCP stars in stellar systems are less frequent. In paper [16] the authors found the rate of binaries with $\mathrm{mCP}$ components as low as $43 \%$ with apparent lack of magnetic objects in eclipsing binaries. With improvements in observational technique, the number of the known eclipsing magnetic system has grown slightly, but it still far from that for the nonmagnetic systems. Avoidance of short-periodic systems is a landmark of magnetic CP stars. Marked differences might be caused by the presence of a strong magnetic field during early evolutional stages and thus they deserve for a detailed study.

The multiplicity of magnetic CP stars has been studied for many years within the individual projects like BinaMIcS [17] and in common surveys like those were carried out in the Special Astrophysical Observatory [18, 19].

\section{Magnetic Measurements}

The Zeeman effect in spectra is the most reliable way for detection and measure of a stellar magnetic field. In the presence of the field, spectral lines split into polarised components. The split pattern depends on the strength of the field, its orientation, and a configuration of each particular atomic transition. The polarisation of the components depends on field geometry and spatial orientation of the star. The Zeeman splitting in non-polarised spectra is only observed in the stars with a strong field and slow axial rotation. That is why spectropolarimetry is proved to be a powerful tool of weak field detection in rotationally broadened spectra. As a comprehensive introductory course on stellar spectropolarimetry we can recommend the reviews written by D. Landstreet [20] and S. Bagnulo [21].

From spectropolarimetric observations one can possible to measure the mean longitudinal field $\left\langle B_{\mathrm{z}}\right\rangle$ which is the line-of-sight component of magnetic field integrated over the visible disc of the star. The distance $\Delta \lambda$ from split circularly-polarised $\sigma$-components to the position $\lambda_{0}$ of an undistorted line depends on the longitudinal field $\left\langle B_{\mathrm{Z}}\right\rangle$ and the Landé factor of the line $g_{\mathrm{L}}$ as follows

$$
\Delta \lambda= \pm 4.67 \cdot 10^{-13} g_{\mathrm{L}} \lambda_{0}^{2}\left\langle B_{\mathrm{z}}\right\rangle .
$$

Wavelength here is measured in angstroms, the mean longitudinal field is in gauss.

If the magnetically split components are visible in unpolarized spectra, one can measure the mean surface magnetic field strength $\langle B\rangle$. This parameter is defined as a disc-integrated modulus of the field strength.

Practically, the Zeeman splitting rarely looks like a simple triplet. For most of the lines, $\pi$ and $\sigma$-components have a complex asymmetric structure that complicates their measuring. 


\section{Three Examples of Multiple Systems}

In this section, we present three magnetic CP stars in binary and multiple systems the study of which may help to shed light on the origin, evolution, and other aspects of the stellar magnetism. The magnetic field of these stars was found with the use of the Russian 6-m telescope BTA.

\subsection{HD 6757}

The magnetic field of HD 6757 was measured for the first time by Kudryavtsev et al. [22]. As the star shows a constantly positive field which varies within a small range, for a long time it has been considered as a slow rotator. Research of more than 15 individual measures of the longitudinal magnetic field does not detect any unambiguous periodicities.

In high-resolution echelle spectra of the star, the line of Fe II $6149 \AA$, which is a standard tool of the magnetic field diagnostic in unpolarized data [23], displays the Zeeman splitting corresponding to $\langle B\rangle=9.9 \mathrm{kG}$. The Nd III $6145 \AA$ line with a triplet-like Zeeman pattern suggests $\langle B\rangle$ closer to $11 \mathrm{kG}$ (Fig. 1). Like the longitudinal field, the mean modulus does not change significantly, which is typical for the slowly-rotating and pole-on stars.

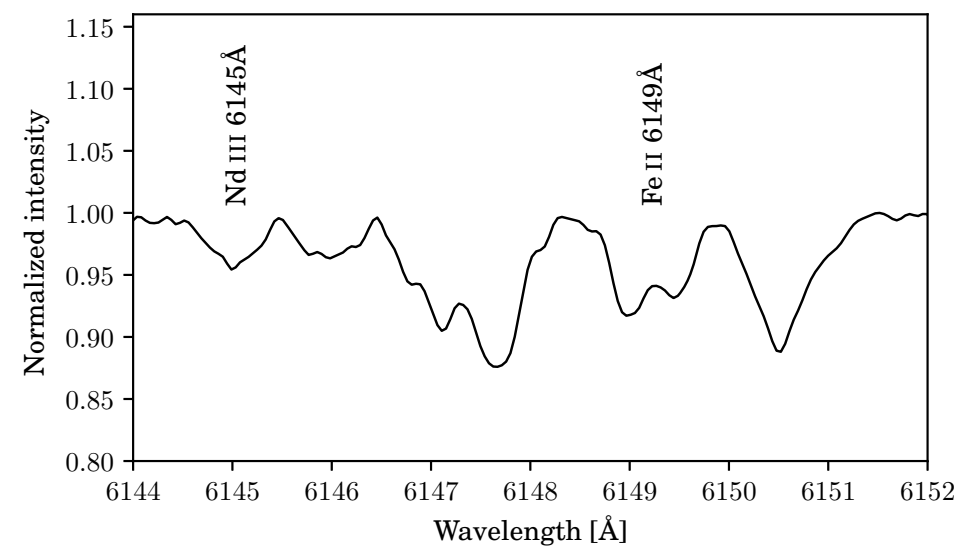

Figure 1. A portion of an echelle spectrum of HD 6757 showing the Zeeman splitting caused by the magnetic field.

We have extracted available TESS $^{1}$ photometry to check for periodic variability. At first glance, the TESS lightcurve contains two signals with periods 9.205 and 1.80098 days (Fig. 2), but the fine detrending is required. Until then, it is impossible to distinguish the sources of the signals.

HD 6757 is a well-known member of a visually binary system ADS 936. At the same time, the echelle spectra of this star obtained in 2006 and 2008 display the trace of at least one more star in hydrogen lines (Fig. 3). The number of available high-resolution spectra is still insufficient to estimate the orbit of this system. The measured radial velocity of the weak spectroscopic component $\left(R V=40-50 \mathrm{~km} \mathrm{~s}^{-1}\right.$ in the red wing of $\mathrm{H}_{\beta}$ line) does not exclude a short orbital period of the spectroscopic system. Thus, we expect that the 9-days period may reflect the orbital motion in the inner spectroscopic system. 


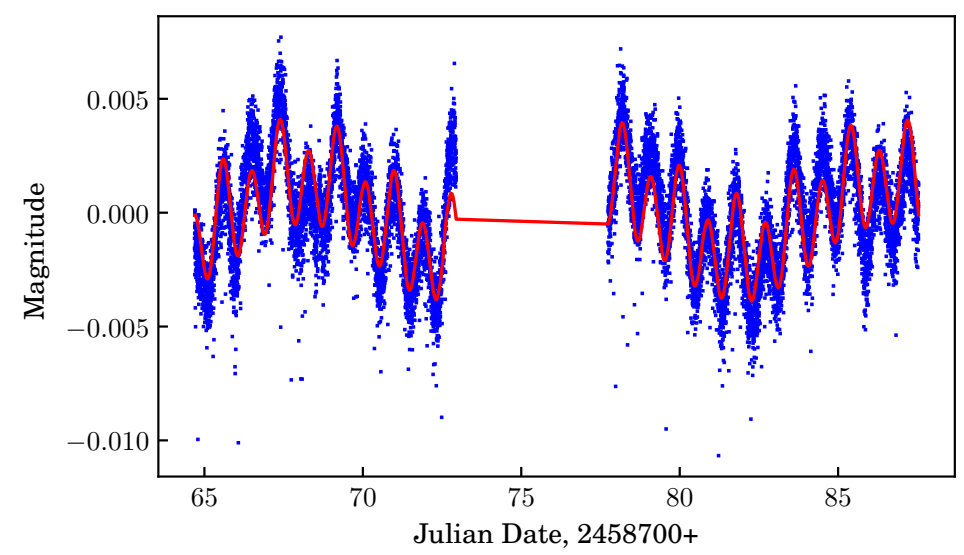

Figure 2. TESS photometry of HD 6757 and its fit with a combination of two periodic functions.

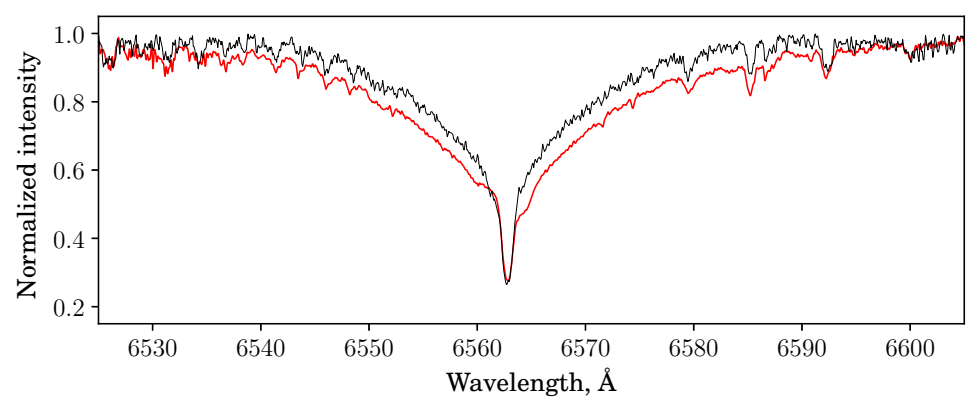

Figure 3. Hydrogen line $\mathrm{H}_{\beta}$ in HD 6757 spectra. The deformation of the transition between the core and wings of the line is clearly seen.

The shorter period could arise from the axial rotation of the CP component. Given $7 \mathrm{~km} \mathrm{~s}^{-1}$ as a projected rotational velocity measured from the spectra, and $R=2 R_{\odot}$ as the most probable radius corresponding to the spectral type, from the oblique rotator model [24] we can expect the dipolar magnetic field with $B_{\mathrm{p}} \approx 13 \mathrm{kG}$, in good agreement with observations.

In the future, we plan to decompose this multiple-star system into components and estimate their physical parameters. That will help to understand how the multiple hierarchical systems evolve.

\subsection{HD 34736}

This 7.82 mag CP star with enhanced lines of silicon in a spectrum was recognized as a magnetic star in 2013 [25]. In observations, the longitudinal field varies from -6 to $+6 \mathrm{kG}$ with a period of 1.2799 days (Fig. 4). This value corresponds to the rotational period of HD 34736,

\footnotetext{
${ }^{1}$ This paper includes data collected by the TESS mission. Funding for the TESS mission is provided by the NASA Explorer Program.
} 
which dominates in TESS photometry (Fig. 5). The original lightcurve was decomposed by Z. Mikulášek onto two components. The first extracted curve has a complex shape typical for CP stars. The second curve also has a complex shape, but its period is much shorter only 0.52 days. The origin of the second curve is still unclear because it might reflect both extremely fast axial rotation of a companion star and pulsation of either component.

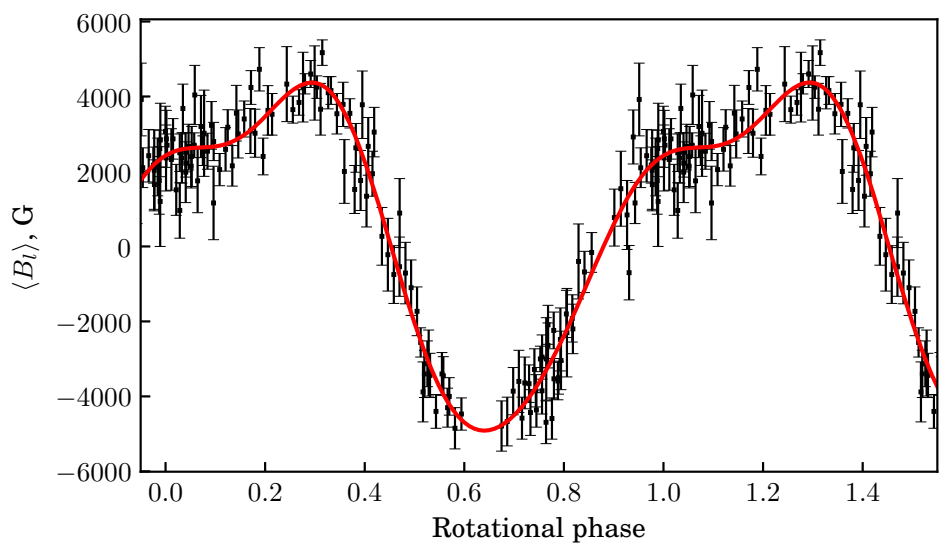

Figure 4. The longitudinal magnetic field of HD 34736 phased with its rotational period.

HD 34736 is a spectroscopic binary system consisting of a magnetic star with relatively narrow lines in the spectrum and a broad-lined star without a measurable magnetic field. Both components are of late B type. With this respect, it is important to establish the real source of the fast periodicity. In the case of fast-rotating secondary star, its variability may suggest the spotted surface and consequently abnormal chemical composition. In this respect, the secondary star by its properties might resemble the well-known $\mathrm{mCP}$ variable CU Vir [26]. Alternatively, the spectral types of both components cannot exclude the pulsation of SPBtype like in the wide binary system $o$ Lup [27]. Either scenario looks possible and is essential for understanding the evolution of binary systems.

All the results of the current study will appear in a dedicated paper.

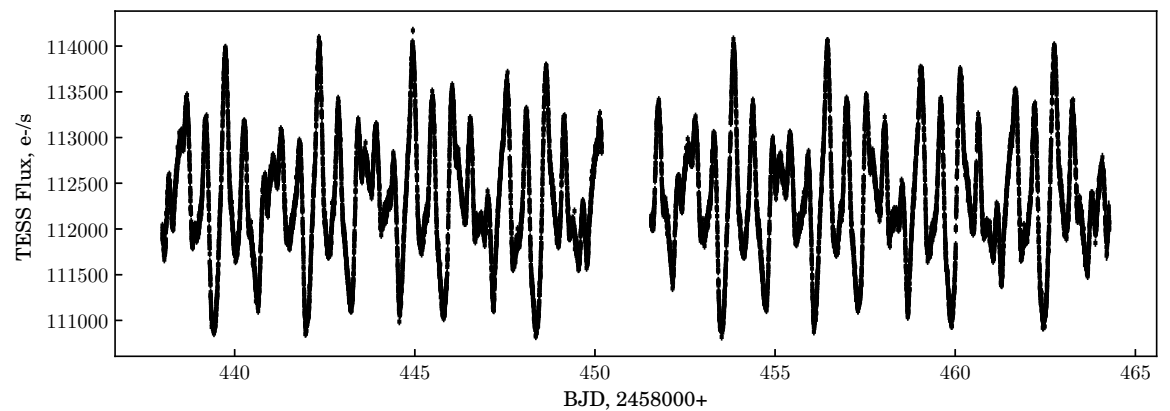

Figure 5. TESS photometry of HD 34736. 


\subsection{HD 40759}

Like in the case of HD 6757, the magnetic field of HD 40759 was discovered by Kudryavtsev et al. [22]. Later this star was included in a spectropolarimetric survey of stars in the Orion OB1 association as HD 40759 is a known member of its subgroup $c$ [28]. During the full period of observation, we have collected more than ten individual measurements of $\left\langle B_{l}\right\rangle$, although the period of its variability remains unclear.

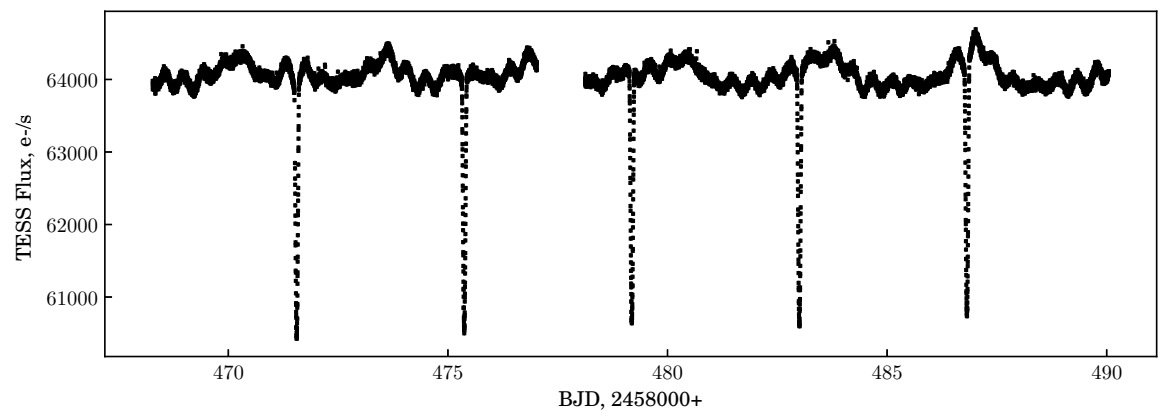

Figure 6. TESS photometry of HD 40759.

The lightcurve of TESS photometry has a waving shape with explicitly seen eclipses (Fig. 6). From an empiric analysis of photometric series, we have derived at least four periodic signals. The system does not exhibit secondary eclipses, and the time between the deep minima (eclipses) is 3.815 days. The second-longest period of 3.432 days may reflect the rotation of the magnetic component, but it cannot fit the measured field $\left\langle B_{l}\right\rangle$. The two other periods are 0.392 and 0.461 days. The origin of the short periodic signals is unclear. It is unlikely that this type of variability comes from rotation. Instead, it might be expected in the case of pulsation. This hypothesis finds support from the fact of stellar multiplicity. HD 40759 is a spectroscopically triple SB3 system as it follows from spectra, obtained with the Russian 6-m telescope. From the measured radial velocities of weaker components and assuming the orbital period equals to time between eclipses, we have tried to reconstruct a spectroscopic orbit of the system. Because of the small number of observations with resolved components, at this point we can find only a set of possible solutions which suggest the elongated orbit with $e=0.4-0.5$ and minimal projected masses $M \sin ^{3} i$ of the components around $2 M_{\odot}$ (Fig. 7). Such combination lets us suppose that the fainter stars are either a pair of two $\delta$ Sct or SPB-type pulsators, depending on the inclination of the orbit. The magnetic CP star is orbiting the inner pair with a very long and undefined period.

\section{Summary}

The three examples from this paper show that the occurrence of magnetic CP stars in binary and multiple systems might be higher than it was commonly believed. Concerning spectroscopically binary stars, one needs to accept that the observational selection is underestimated, first of all, due to the low number of corresponding observations per one system. The examples of HD 6757 and HD 34736 perfectly demonstrate the value of a large number of high-resolution spectra. Both stars do not expose the lines of all components most of the time. 

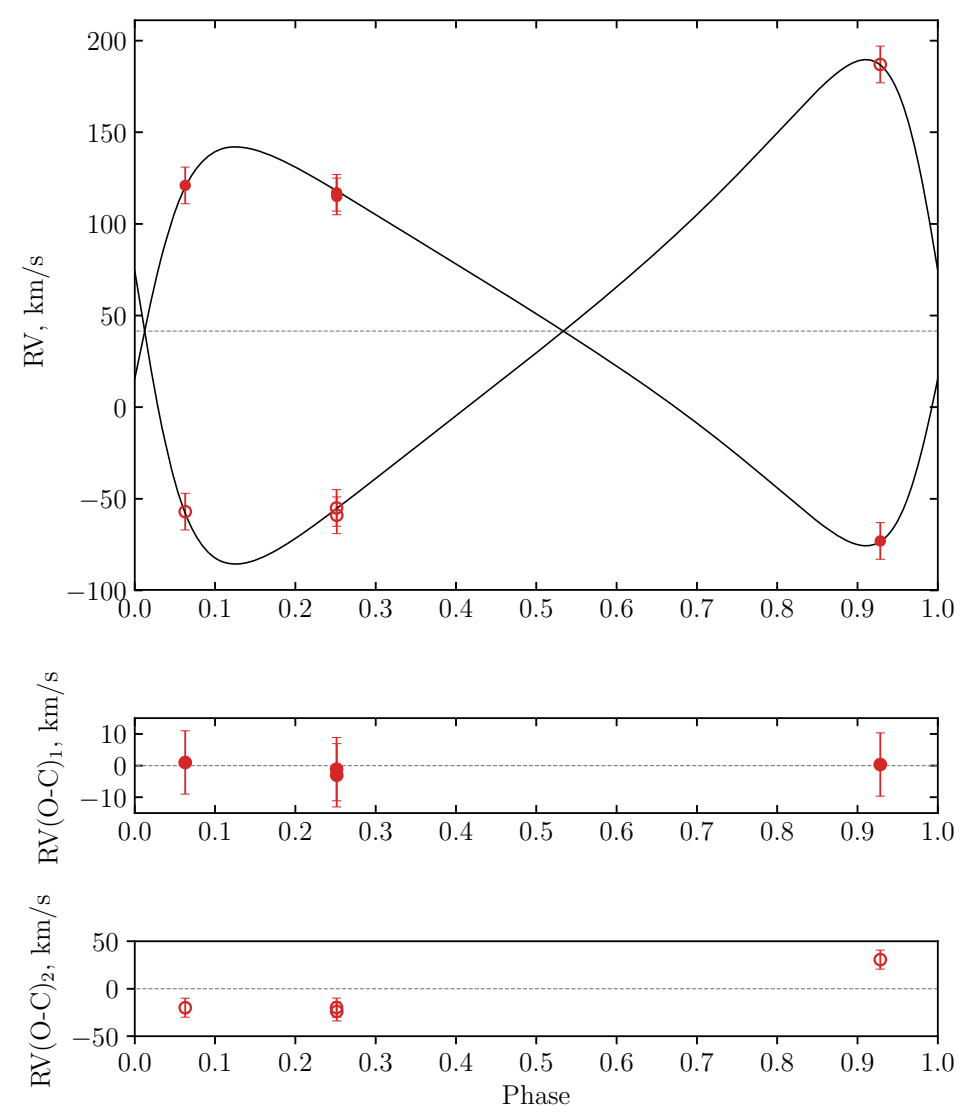

Figure 7. Possible orbital solution for HD 40759.

In searches of new interesting binary systems, good results can be achieved when the high-resolution spectroscopy combines with photometry. Time-resolved data obtained by the TESS satellite suit perfectly for such purposes. Solely from photometry one can find the pulsating components in the systems like HD 34736 or HD 40759 which are too faint for time-resolved and high-resolution spectroscopy. The fact of the presence of pulsating stars in the system is especially important because depending on the amplitude and period, it allows putting the restrictions on expected physical parameters of weak companions. HD 40759, in this sense, appears as a unique laboratory.

All the results presented in this paper are preliminary. For the stars HD 6757 and HD 40759 in an observational season 2019/2020 with the 2.4-m Thai National Telescope we have collected a substantial set of echelle spectra which would be included in the research. After all, the results will be published in the relevant papers.

Acknowledgements. The author is thankful to his colleagues I. Romanyuk, I. Yakunin, A. Moiseeva (all from the Special Astrophysical Observatory, Russia), Z. Mikulášek (Masaryk University, Czech Republic), and D. Mkrtichian (NARIT, Thailand) for their valuable help in collecting and analysis of observational data. 


\section{References}

[1] L. Ferrario, Contributions of the Astronomical Observatory Skalnate Pleso 48, 15 (2018), 1801.09368

[2] D. Shulyak, A. Reiners, E. Nagel, L. Tal-Or, J.A. Caballero, M. Zechmeister, V.J.S. Béjar, M. Cortés-Contreras, E.L. Martin, A. Kaminski et al., Astronomy and Astrophysics 626, A86 (2019), 1904 . 12762

[3] P. Mathias, M. Aurière, A. López Ariste, P. Petit, B. Tessore, E. Josselin, A. Lèbre, J. Morin, G. Wade, F. Herpin et al., Astronomy and Astrophysics 615, A116 (2018), 1804.01831

[4] B. Das, P. Chandra, M.E. Shultz, G.A. Wade, Monthly Notices of the RAS 489, L102 (2019), 1908.09110

[5] L. Mestel, J.D. Landstreet, Stellar Magnetic Fields (2005), Vol. 664, p. 183

[6] L. Mestel, D. Moss, Monthly Notices of the RAS 405, 1845 (2010)

[7] E.F. Borra, J.D. Landstreet, L. Mestel, Annual Review of Astron and Astrophys 20, 191 (1982)

[8] C. Neiner, S. Mathis, E. Alecian, C. Emeriau, J. Grunhut, BinaMIcS, MiMeS Collaborations, The origin of magnetic fields in hot stars, in Polarimetry, edited by K.N. Nagendra, S. Bagnulo, R. Centeno, M. Jesús Martínez González (2015), Vol. 305 of IAU Symposium, pp. 61-66, 1502.00226

[9] L. Ferrario, J.E. Pringle, C.A. Tout, D.T. Wickramasinghe, Monthly Notices of the RAS 400, L71 (2009)

[10] G.W. Preston, Annual Review of Astron and Astrophys 12, 257 (1974)

[11] N. Piskunov, O. Kochukhov, Astronomy and Astrophysics 381, 736 (2002)

[12] O. Kochukhov, arXiv e-prints arXiv:1912.07241 (2019), 1912.07241

[13] V.D. Bychkov, L.V. Bychkova, J. Madej, Monthly Notices of the RAS 394, 1338 (2009)

[14] S. Hubrig, J. González, M. Schöller, Multiplicity of Chemically Peculiar Stars (2010), Vol. 435 of Astronomical Society of the Pacific Conference Series, p. 257

[15] M. Schöller, S. Correia, S. Hubrig, N. Ageorges, Astronomy and Astrophysics 522, A85 (2010), 1010.3643

[16] F. Carrier, P. North, S. Udry, J. Babel, Astronomy and Astrophysics 394, 151 (2002), astro-ph/0208082

[17] E. Alecian, C. Neiner, G.A. Wade, S. Mathis, D. Bohlender, D. Cébron, C. Folsom, J. Grunhut, J.B. Le Bouquin, V. Petit et al., The BinaMIcS project: understanding the origin of magnetic fields in massive stars through close binary systems, in New Windows on Massive Stars, edited by G. Meynet, C. Georgy, J. Groh, P. Stee (2015), Vol. 307 of IAU Symposium, pp. 330-335, 1409. 1094

[18] Y.Y. Balega, V.V. Dyachenko, A.F. Maksimov, E.V. Malogolovets, D.A. Rastegaev, I.I. Romanyuk, Astrophysical Bulletin 67, 44 (2012)

[19] E.A. Semenko, I.I. Romanyuk, I.A. Yakunin, A.V. Moiseeva, D.O. Kudryavtsev, Binary Stars in the Orion OB1 Association, Subgroup a (2019), Vol. 518 of Astronomical Society of the Pacific Conference Series, p. 31

[20] J.D. Landstreet, Basics of spectropolarimetry, in New Windows on Massive Stars, edited by G. Meynet, C. Georgy, J. Groh, P. Stee (2015), Vol. 307 of IAU Symposium, pp. 311320

[21] M.I. Mishchenko, Y.S. Yatskiv, V.K. Rosenbush, G. Videen, Polarimetric Detection, Characterization and Remote Sensing (2011) 
[22] D.O. Kudryavtsev, I.I. Romanyuk, V.G. Elkin, E. Paunzen, Monthly Notices of the RAS 372, 1804 (2006)

[23] G. Mathys, S. Hubrig, J.D. Landstreet, T. Lanz, J. Manfroid, Astronomy and Astrophysics Supplement 123, 353 (1997)

[24] J.J. Monaghan, Monthly Notices of the RAS 163, 423 (1973)

[25] E.A. Semenko, I.I. Romanyuk, D.O. Kudryavtsev, I.A. Yakunin, Astrophysical Bulletin 69, 191 (2014), 1404. 5937

[26] R. Kuschnig, T.A. Ryabchikova, N.E. Piskunov, W.W. Weiss, M.J. Gelbmann, Astronomy and Astrophysics 348, 924 (1999)

[27] B. Buysschaert, C. Neiner, A.J. Martin, M.E. Oksala, C. Aerts, A. Tkachenko, E. Alecian, MiMeS Collaboration, Astronomy and Astrophysics 622, A67 (2019)

[28] I.I. Romanyuk, E.A. Semenko, I.A. Yakunin, D.O. Kudryavtsev, Astrophysical Bulletin 68, 300 (2013) 